\title{
Improved detection of long-range residual dipolar couplings in weakly aligned samples by Lee-Goldburg decoupling of homonuclear dipolar truncation
}

\author{
Pernille Jensen, Hans-Jürgen Sass \& Stephan Grzesiek* \\ Division of Structural Biology, Biozentrum der Universität Basel, Klingelbergstrasse 70, CH-4056 Basel, \\ Switzerland
}

Received 24 September 2004; Accepted 18 October 2004

Key words: NMR, protein, structure, weak alignment

\begin{abstract}
Homonuclear ${ }^{1} \mathrm{H}$ residual dipolar couplings (RDCs) truncate the evolution of transverse ${ }^{1} \mathrm{H}$ magnetization of weakly aligned molecules in high-resolution NMR experiments. This leads to losses in sensitivity or resolution in experiments that require extended ${ }^{1} \mathrm{H}$ evolution times. Lee-Goldburg decoupling schemes have been shown to remove the effects of homonuclear dipolar couplings, while preserving chemical shift evolution in a number of solid-state NMR applications. Here, it is shown that the LeeGoldburg sequence can be effectively incorporated into INEPT- or HMQC-type transfer schemes in liquid state weak alignment experiments in order to increase the efficiency of the magnetization transfer. The method is applied to the sensitive detection of ${ }^{1} \mathrm{H}^{\mathrm{N}}-{ }^{13} \mathrm{C}$ long-range RDCs in a three-dimensional $\mathrm{HCN}$ experiment. As compared to a conventional $\mathrm{HCN}$ experiment, an average sensitivity increase by a factor of 2.4 is obtained for a sample of weakly aligned protein $\mathrm{G}$. This makes it possible to detect 170 long-range ${ }^{1} \mathrm{H}^{\mathrm{N}}-{ }^{13} \mathrm{C}$ RDCs for distances up to $4.9 \AA$.
\end{abstract}

\section{Introduction}

Residual tensorial couplings of weakly aligned molecules have become an invaluable source of structural and dynamical information in high resolution NMR (Tolman et al., 1995; Tjandra and Bax, 1997; Bax, 2003). Most commonly, one-bond residual dipolar couplings (RDCs) are used as the most sensitive and easy interpretable parameters. However, recently also a number of experiments have been proposed for the detection of long-range RDCs (Wu and Bax, 2002; Boisbouvier et al., 2003; Meier et al., 2003). Similar to long-range NOEs, such long-range RDCs carry very valuable information on structure,

*To whom correspondence should be addressed. E-mail: Stephan.Grzesiek@unibas.ch long-range order, and dynamics of biomolecules that is not easily accessible from short-range interactions.

The sensitivity of such long-range RDC experiments is limited by the $1 / r^{3}$ dependence of the dipolar coupling size, the transverse relaxation times, and by coherent broadening from multiple residual dipolar couplings. In particular, multiple dipolar couplings among protons severely truncate the transverse evolution of proton magnetization and limit the detectable distances in long-range RDC experiments from protons. Perdeuteration followed by reprotonation of labile hydrogen positions (LeMaster and Richards, 1988; Torchia et al., 1988) greatly alleviates this problem for the observation of long-range RDCs from amide protons $\left({ }^{1} \mathrm{H}^{\mathrm{N}}\right)$ to surrounding ${ }^{1} \mathrm{H}^{\mathrm{N}}(\mathrm{Wu}$ and $\mathrm{Bax}, 2002$; Meier 
et al., 2003) and ${ }^{13} \mathrm{C}$ nuclei (Meier et al., 2003). However, the dipolar couplings to the remaining population of nearby ${ }^{1} \mathrm{H}^{\mathrm{N}}$ nuclei, e.g., in $\alpha$-helices, still limits the ${ }^{1} \mathrm{H}^{\mathrm{N}}$ linewidth or effective transverse magnetization lifetime for small to medium-sized aligned proteins. This effect reduces the sensitivity of long-range RDC experiments from amide protons to heteronuclei, which use classical INEPT of HMQC transfers.

Continuous wave $(\mathrm{CW})$ off-resonance irradiation around the magic angle as proposed by Lee and Goldburg (Lee and Goldburg, 1965) is a common technique for decoupling of homonuclear dipolar interactions in solid state NMR. The rotation of spins around the magic angle effectively averages out the homonuclear dipolar coupling Hamiltonian, while scaling chemical shift terms and heteronuclear scalar or dipolar couplings by a factor of $1 / \sqrt{ } 3$. A considerable improvement of the performance of LG decoupling is achieved by frequency switching (FSLG) (Mehring and Waugh, 1972; Bielecki et al., 1989) or equivalently (and less demanding on the frequency switching capabilities of NMR spectrometers) by phase modulation (PMLG) (Vinogradov et al., 1999).

The efficiency of the PMLG decoupling has been shown in 2D studies of solid samples (Vinogradov et al., 1999) and simple organic molecules dissolved in nematic liquid crystalline solvents (Marjanska et al., 2002). Here, we show that PMLG decoupling can also be used very effectively to eliminate the effects of residual dipolar truncation in liquid state experiments on weakly aligned biomolecules and that PMLG can be easily incorporated into INEPT and HMQC transfer schemes. This leads to significant sensitivity increases for the detection of heteronuclear long-range RDCs from amide protons in weakly aligned perdeuterated proteins.

\section{Materials and methods}

NMR samples consisted of $2.22 \mathrm{mM}$ uniformly ${ }^{15} \mathrm{~N} /{ }^{13} \mathrm{C} /{ }^{2} \mathrm{H}$-labelled (amide protonated) protein $\mathrm{G}, \mathrm{pH} 5.6,95 \% \mathrm{H}_{2} \mathrm{O} / 5 \% \mathrm{D}_{2} \mathrm{O}$ either aligned by the presence of $30 \mathrm{mg} / \mathrm{ml}$ filamentous phages Pf1 (Hansen et al., 1998) or under isotropic conditions in the absence of phages. Due to use for protein engineering, the sequence of this protein
G construct slightly deviates from the published X-ray structure 1IGD (Derrick and Wigley, 1994). The current sequence is ${ }^{6} \mathrm{MQYKL}{ }^{11} \mathrm{AL}-$ NGKTLKGE ${ }^{21}$ TTTEAVDAAT ${ }^{31}$ AEKVFKQYAN ${ }^{41}$ DNGVDGEWTY ${ }^{51}$ DDATKTFTVT ${ }^{61}$ ELVPRGSIEGR using the 1IGD numbering scheme. All NMR experiments were carried out at $25^{\circ} \mathrm{C}$ on a Bruker DRX-600 spectrometer, equipped with a triple resonance, 3-axis pulsed field gradient probe.

The PMLG decoupling sequence was implemented by shaped pulses according to the phase modulation scheme described by Vega and coworkers (Vinogradov et al., 1999). For this, one LG-unit of length $t_{\mathrm{LG}}$ was divided into $m$ constant-phase units such that the rf field Hamiltonian takes the form

$$
\begin{aligned}
& H_{\mathrm{rf}}=\omega_{1}\left[I_{x} \cos \left(\Delta \alpha\left(i+\frac{1}{2}\right)\right)+I_{y} \sin \left(\Delta \alpha\left(i+\frac{1}{2}\right)\right)\right] \\
& \omega_{1}=\sqrt{\frac{2}{3}} \frac{2 \pi}{t_{\mathrm{LG}}}=\sqrt{2}\left|\omega_{\mathrm{LG}}\right|
\end{aligned}
$$

with $i=0,1, \ldots, m-1$ and $\Delta \alpha=\alpha_{\mathrm{LG}} / m$, where $\alpha_{\mathrm{LG}}=2 \pi / \sqrt{ } 3=207.8^{\circ}$ is the accumulated phase for one LG-unit. This rf field Hamiltonian corresponds approximately to a CW irradiation of field strength $\omega_{1}$ at resonance offset $\omega_{\mathrm{LG}}$. The LG-unit (denoted by $X$ ) is then followed by a phase-inverted $\mathrm{CW}$ irradiation at offset $-\omega_{\mathrm{LG}}$ (denoted by $\bar{X}$ ), which was approximated in the pulse-programming unit as

$$
\begin{aligned}
H_{\mathrm{rf}}=\omega_{1}[ & I_{x} \cos \left(\Delta \alpha\left(2 m-\frac{3}{2}-i\right)\right) \\
& \left.\quad+I_{y} \sin \left(\Delta \alpha\left(2 m-\frac{3}{2}-i\right)\right)\right]
\end{aligned}
$$

for $i=m, m+1, \ldots, 2 m-1$. In praxis, the $\mathrm{LG}$ irradiations consisted of an integer number of supercycles $X \overline{X X} X$, the number of phase increments $m$ was set to 10 , and an rf field strength of $\omega_{1}=2 \pi \cdot 7.5 \mathrm{kHz}$ was used, such that $t_{\mathrm{LG}}=$ $108.9 \mu \mathrm{s}$.

It should be noted that the required rf field strength for the LG irradiation is considerably lower than in typical solid state applications, since $\omega_{1}$ needs only to be large as compared to the chemical shift differences and the residual dipolar couplings, but not relative to the full solid state dipolar powder pattern width. The applied field strength of $2 \pi \cdot 7.5 \mathrm{kHz}$ and the 
length of the irradiation in the current experiments are thus comparable to normal ${ }^{1} \mathrm{H}-{ }^{1} \mathrm{H}$ TOCSY experiments and still considerably smaller than the expected safe limits for our BRUKER TXI probes $(2 \pi \cdot 10 \mathrm{kHz}, 300 \mathrm{~ms})$.

Data were processed and analyzed with the NMRPipe suite of programs (Delaglio et al., 1995). Coupling constants were derived from the $3 \mathrm{D}$ cross or $2 \mathrm{D}$ reference long-range $\mathrm{HCN}$ experiments as described previously (Meier et al., 2003). In brief, the coupling constant $K_{\mathrm{HC}}$ was extracted from the ratio of cross and reference peak intensities of the $\mathrm{HCN}$-experiment in the following way: peaks of the cross experiment were fitted by the time domain fitting routine nlinLS contained in the NMRPipe package such that the amplitudes $\left(A_{\mathrm{c}}\right)$ of the $t_{1}\left({ }^{13} \mathrm{C}\right)$-FIDs were obtained. The amplitude of the reference signal $\left(A_{\mathrm{R}}\right)$ was obtained from an analogous fit. The amplitudes were corrected for the different numbers of acquired scans in both experiments. $\left|K_{\mathrm{HC}}\right|$ was then calculated from the ratio $A_{\mathrm{C}} / A_{\mathrm{R}}$ of both amplitudes as $\left|K_{\mathrm{HC}}\right|=\operatorname{atan}\left(\left|A_{\mathrm{C}} / A_{\mathrm{R}}\right|^{1 / 2}\right) /$ $\left(\pi T_{\mathrm{LG}} / \sqrt{ } 3\right)$.

\section{Results and discussion}

\section{Efficiency of PMLG homonuclear dipolar decoupling}

In order to demonstrate the efficiency of the homonuclear dipolar decoupling by the PMLG sequence, a ${ }^{1} \mathrm{H}^{\mathrm{N}}$ spin-echo experiment with subsequent detection of amide resonances by a ${ }^{1} \mathrm{H}^{\mathrm{N}}{ }^{15} \mathrm{~N}$ HSQC was carried out on a sample of Pf1-phage oriented $\left({ }^{1} \mathrm{D}_{\mathrm{HN}, \max }=26 \mathrm{~Hz}\right)$ uniformly ${ }^{15} \mathrm{~N} /{ }^{13} \mathrm{C} /{ }^{2} \mathrm{H}$-labelled (amide protonated) protein $\mathrm{G}$ (Figure 1). During the free precession delays of the spin echo, PMLG decoupling was either applied $(+\mathrm{LG})$ or not applied (-LG). For short spin-echo periods of few milliseconds the intensities of the + LG and -LG spin-echo experiments were virtually identical (data shown). Figure 1 depicts data for the decay of the amide ${ }^{1} \mathrm{H}^{\mathrm{N}}$ magnetization for residues R65 and N40 during the two experiments. Since the evolution of ${ }^{1} \mathrm{H}^{\mathrm{N}}$ chemical shifts and heteronuclear couplings is slowed down by $1 / \sqrt{3}$ during the Lee-Goldburg magic angle spin lock, for a fair comparison the respective spin echo delays for the PMLG

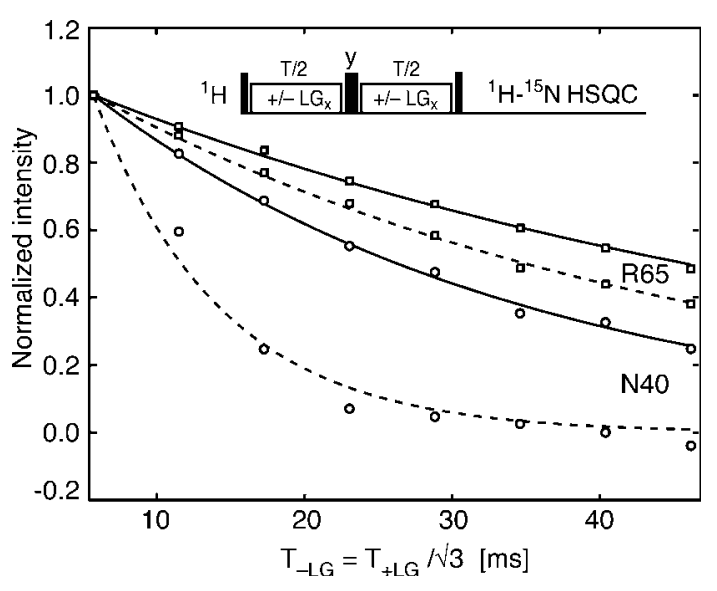

Figure 1. Efficiency of ${ }^{1} \mathrm{H}-{ }^{1} \mathrm{H}$ dipolar decoupling by $\mathrm{PMLG}$ in weakly aligned protein $\mathrm{G}$. Data points correspond to resonance intensities measured by a ${ }^{1} \mathrm{H}^{15} \mathrm{~N}$ HSQC after a ${ }^{1} \mathrm{H}$ spin-echo experiment with ( $+\mathrm{LG}$, solid lines) or without $(-\mathrm{LG}$, broken lines) PMLG decoupling in both $T / 2$ periods (see inset). Since chemical shifts and heteronuclear scalar coupling are decreased by $\sqrt{ } 3$ during $L G$ decoupling, the horizontal axis corresponds to the delay time $\mathrm{T}$ without $\mathrm{LG}$ decoupling or to $T / \sqrt{3}$ with $\mathrm{LG}$ decoupling. Data for residue R65 and N40 are shown as open squares and open circles, respectively. Intensities are normalized to the first measured point at $T_{-\mathrm{LG}}=5.7 \mathrm{~ms}$. The inset shows the schematics of the ${ }^{1} \mathrm{H}-{ }^{15} \mathrm{~N}$ HSQC-detected ${ }^{1} \mathrm{H}$ spinecho pulse sequence.

decoupling scheme $(+\mathrm{LG})$ were increased in length by $\sqrt{ } 3$ relative to the (-LG) experiment. It is evident from Figure 1 that, even when using this different scaling of the spin echo periods, the decay of transverse ${ }^{1} \mathrm{H}^{\mathrm{N}}$ magnetization is slowed down by the PMLG decoupling. Thus for residue R65 located in the flexible C-terminus of our protein $\mathrm{G}$ construct the decay time increases from 41 to $55 \mathrm{~ms}$. Similar increases are found for most other residues (data not shown), whereas even stronger increases in the decay times are found for residues located in the $\alpha$-helix of protein $\mathrm{G}$. Thus, for example residue N40 shows an increase in the apparent decay time from 9 to $33 \mathrm{~ms}$ (Figure 1). This is consistent with the fact that the strongest ${ }^{1} \mathrm{H}^{\mathrm{N}}-{ }^{1} \mathrm{H}^{\mathrm{N}}$ residual dipolar couplings are found in the $\alpha$-helix due to the short ${ }^{1} \mathrm{H}^{\mathrm{N}}-{ }^{1} \mathrm{H}^{\mathrm{N}}$ distances and a helix orientation, where many of the ${ }^{1} \mathrm{H}^{\mathrm{N}}-{ }^{1} \mathrm{H}^{\mathrm{N}}$ vectors have similar directions as the principal axes of the alignment tensor with the largest or smallest eigenvalues.

It should be noted that the transverse relaxation rate $\mathrm{R}_{2, \mathrm{LG}}$ for magnetization that is rotating within the tilted Lee-Goldburg frame, i.e., perpendicular to the magic angle $\left(\theta_{\mathrm{m}}\right)$, is given by 
$R_{2, \mathrm{LG}}=\sin ^{2} \theta_{\mathrm{m}} \cdot R_{2}+\cos ^{2} \theta_{\mathrm{m}} \cdot R_{1}=2 / 3 \cdot R_{2}$ $+1 / 3 \cdot R_{1}$, where $R_{2}$ and $R_{1}$ are the transverse and longitudinal relaxation rates in the untilted frame. Thus for $R_{1} \ll R_{2}$, the relative reduction in $\mathrm{R}_{2, \mathrm{LG}}$ is slightly smaller $(\approx 0.67)$ than the scaling of the chemical shift and heteronuclear coupling evolution $(1 / \sqrt{ } 3 \approx 0.58)$. As evident from the data in Figure 1, the loss from this slightly faster effective transverse relaxation during the PMLG sequence is overcompensated by the suppression of the homonuclear dipolar truncation.

Another point of interest is the strong influence of $\mathrm{rf}$ field inhomogeneities on the efficiency of the PMLG sequences (Vega, 2004). As the chemical shift evolution occurs in the frame of the effective field, i.e., offset + rf spin-lock, inhomogeneities in the rf field strength cannot easily be compensated. The detrimental effect of such inhomogeneities is clearly detectable, when the $180^{\circ}$ refocusing pulse is omitted from the spin echo sequence in Figure 1 (data not shown). In this case, the free precession oscillations during the spin echo period decay much faster than the refocused signals depicted in Figure 1 . The $180^{\circ}$ pulse in the middle of the PMLG period effectively refocuses the dephasing of magnetization from the rf inhomogeneities such that no signal loss occurs. Simulations of this situation by the program Matlab corroborate these findings. For the implementation of the long-range RDC experiment (see below), the PMLG sequence is used in combination with refocusing $180^{\circ}$ pulses during the INEPT- and HMQC-transfer steps such that losses from rf inhomogeneities are avoided. However, such losses may be important when the PMLG sequence is used during free precession periods, e.g., for the detection of dipolar-decoupled chemical shift evolution in the original PMLG experiment (Vinogradov et al., 1999). Implementation of the PMLG long-range ${ }^{1} H^{N-}{ }^{13} C$
RDC experiment

In order to demonstrate the sensitivity gain that can be obtained by the decoupling of residual homonuclear ${ }^{1} \mathrm{H}$ dipolar interactions, the PMLG decoupling scheme was incorporated into a quantitative J-experiment for the detection of longrange ${ }^{1} \mathrm{H}^{\mathrm{N}}-{ }^{13} \mathrm{C}$ dipolar couplings (Figure 2). This experiment is a variant of the long-range quantitative HCN-HSQC without homonuclear decoup- ling published earlier (Meier et al., 2003). The new three-dimensional experiment is a concatenation of a ${ }^{1} \mathrm{H}^{\mathrm{N}}-{ }^{15} \mathrm{~N}$ HMQC and a quantitative ${ }^{1} \mathrm{H}^{\mathrm{N}}-{ }^{13} \mathrm{C}$ HSQC. In brief, proton magnetization $\mathrm{H}^{\mathrm{N}}$ y present at point $a$ is transferred during the interval $\delta\left[=9.3 \mathrm{~ms} \approx \sqrt{ } 3 /\left(2{ }^{1} J_{\mathrm{HN}}\right)\right]$ by the reduced scalar coupling ${ }^{1} J_{\mathrm{HN}} / \sqrt{ } 3$ into proton-nitrogen multiquantum magnetization $2 \mathrm{H}^{\mathrm{N}}{ }_{x} \mathrm{~N}_{y}$ at point $b$. Subsequently, the multiquantum magnetization is labeled by the ${ }^{15} \mathrm{~N}$ frequency during the constant time evolution period (4T) from points $b$ to $c$. Magnetization is then transferred back from points $c$ to $d$ by the reduced coupling ${ }^{1} J_{\mathrm{HN}} / \sqrt{ } 3$ into transverse proton magnetization, which is inphase with respect to nitrogen. The reduced scalar and dipolar ${ }^{1} \mathrm{H}^{\mathrm{N}}-{ }^{13} \mathrm{C}$ couplings $\left(K_{\mathrm{HC}} / \sqrt{ } 3=\mathrm{J}_{\mathrm{HC}} /\right.$ $\left.\sqrt{3}+\mathrm{D}_{\mathrm{HC}} / \sqrt{3}\right)$ are active in the tilted frame of the magic angle spin lock during the entire $T_{L G}$ period from point $a$ to point $d$. Therefore, the magnetization at point $d$ consists of terms, which are proportional to $\mathrm{H}^{\mathrm{N}} \cdot \cos \left(\pi K_{\mathrm{HC}} T_{\mathrm{LG}} / \sqrt{ } 3\right)$ $-2 \mathrm{H}^{\mathrm{N}}{ }_{X} \mathrm{C}_{z} \cdot \sin \left(\pi K_{\mathrm{HC}} T_{\mathrm{LG}} / \sqrt{ } 3\right)$. In this notation, the $\mathrm{H}^{\mathrm{N}}{ }_{X}$ and $\mathrm{H}^{\mathrm{N}}{ }_{Y}$ terms correspond to components of proton magnetization in the tilted coordinate system of the LG spin-lock, such that $\mathrm{H}^{\mathrm{N}}{ }_{X}$ $=\mathrm{H}^{\mathrm{N}}{ }_{x} \cos \theta_{\mathrm{m}}-\mathrm{H}^{\mathrm{N}} \sin \theta_{\mathrm{m}} ; \mathrm{H}^{\mathrm{N}}{ }_{Y}=\mathrm{H}^{\mathrm{N}} ; \mathrm{H}^{\mathrm{N}}{ }_{Z}$ $=\mathrm{H}^{\mathrm{N}}{ }_{z} \cos \theta_{\mathrm{m}}+\mathrm{H}^{\mathrm{N}}{ }_{x} \sin \theta_{\mathrm{m}}$. For the detection of the ${ }^{1} \mathrm{H}^{\mathrm{N}} \rightarrow{ }^{13} \mathrm{C}$ transfer, the anti-phase term $-2 \mathrm{H}^{\mathrm{N}}{ }_{X} \mathrm{C}_{z} \sin \left(\pi K_{\mathrm{HC}} T_{\mathrm{LG}} / \sqrt{3}\right)$ at point $d$ is converted into anti-phase carbon magnetization $-2 \mathrm{H}_{\mathrm{z}}^{\mathrm{N}} \mathrm{C}_{\mathrm{y}} \cdot \sin \left(\pi K_{\mathrm{HC}} T_{\mathrm{LG}} / \sqrt{ } 3\right)$ at point $e$ by the $\left(90^{\circ}-\theta_{\mathrm{m}}\right)$ flip angle proton $\phi_{3}$-pulse and the $90^{\circ}$ carbon $\phi_{4}$-pulse. This component is selected by phase cycling, and ${ }^{13} \mathrm{C}$ frequency labeling is achieved in the usual manner during the $t_{1}$-delay. Magnetization is then transferred back into observable in-phase ${ }^{1} \mathrm{H}^{\mathrm{N}}$ terms at point $g$ via a reverse PMLG-INEPT and a subsequent WATERGATE (Piotto et al., 1992) module.

The transfer scheme gives rise to three-dimensional $\left(\omega_{\mathrm{C}}, \omega_{\mathrm{N}}, \omega_{\mathrm{HN}}\right)$ signals with intensities proportional to $\sin ^{2}\left(\pi K_{\mathrm{HC}} T_{\mathrm{LG}} / \sqrt{ } 3\right)$. In a manner analogous to the original quantitative $\mathrm{HCN}$ experiment (Meier et al., 2003), $K_{\mathrm{HC}}$ coupling constants are quantified from a comparison to a reference experiment. For this, the phase of the $\phi_{3}$-pulses is changed to select for the $\mathrm{H}_{y^{\prime}} \cdot \cos \left(\pi K_{\mathrm{HC}} T_{\mathrm{LG}} / \sqrt{ } 3\right)$ term at point $d$, the corresponding flip angle is set to $90^{\circ}$, and the $t_{1}$-incrementation is omitted. This spectrum gives rise to two-dimensional $\left(\omega_{\mathrm{N}}, \omega_{\mathrm{HN}}\right)$ signals 


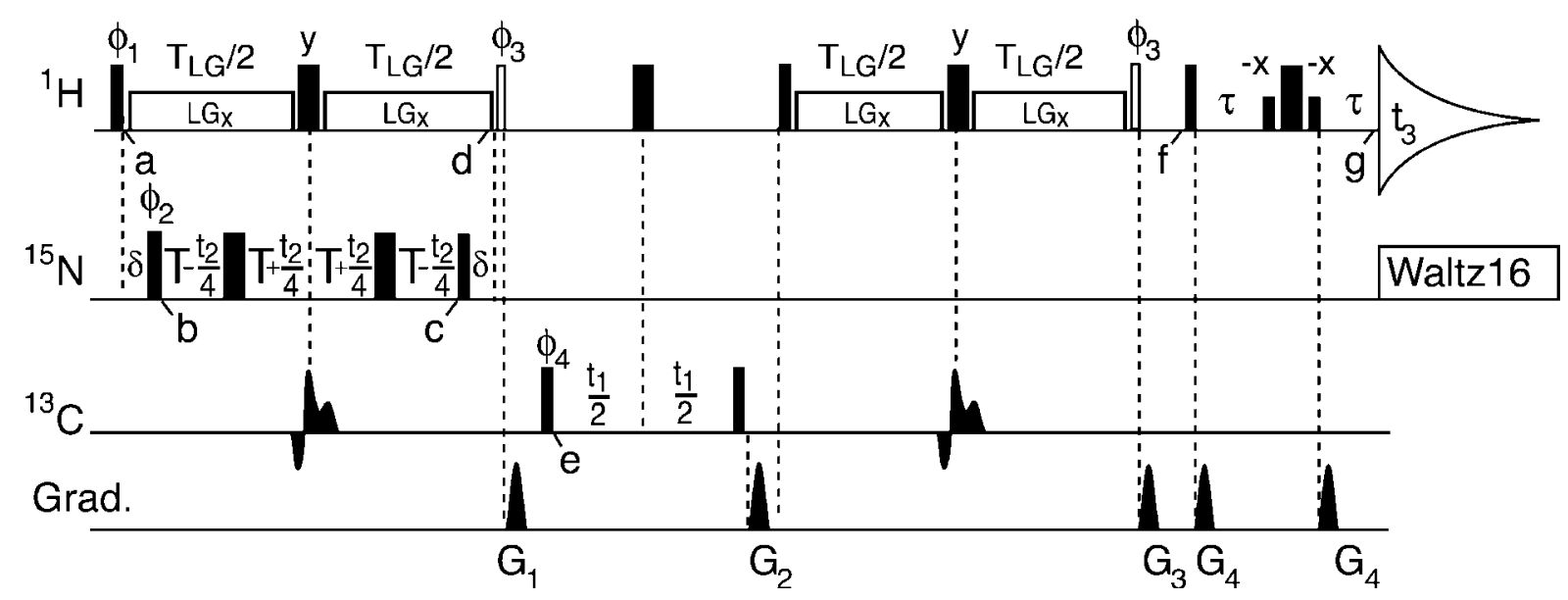

Figure 2. Pulse sequence of the phase modulated Lee-Goldburg $\mathrm{HCN}$ experiment for measurement of long-range ${ }^{1} \mathrm{H}-{ }^{13} \mathrm{C}$ RDCs. Filled narrow and wide pulses denote $90^{\circ}$ and $180^{\circ}$ flip angles respectively. Open bars denote pulses with a flip angle of $\left(90^{\circ}-\theta_{\mathrm{m}}\right)$, where $\theta_{\mathrm{m}}$ is the magic angle. Unless indicated otherwise, the phase is $\mathrm{x}$. Rectangular high (low) power ${ }^{1} \mathrm{H}$ pulses are applied using an $\mathrm{rf}$ field strength of $36(0.25) \mathrm{kHz}$. To obtain maximal efficiency from the limited rf strength of Lee-Goldburg decoupling $(7.5 \mathrm{kHz})$, the ${ }^{1} \mathrm{H}$ carrier is set to $9.5 \mathrm{ppm}$ during the first part of the sequence and switched to the ${ }^{1} \mathrm{H}_{2} \mathrm{O}$ resonance before the WATERGATE module (Piotto et al., 1992) at point $f$. For detection of RDCs between ${ }^{1} \mathrm{H}^{\mathrm{N}}$ and aliphatic ${ }^{13} \mathrm{C}$ nuclei, the carrier frequency for solid shape ${ }^{13} \mathrm{C}$ pulses is set to $43 \mathrm{ppm} .{ }^{13} \mathrm{C} 90^{\circ}$-pulses have an rf strength of $23 \mathrm{kHz}$, whereas the shaped ${ }^{13} \mathrm{C} 180^{\circ} \mathrm{G} 3$-pulses (Emsley and Bodenhausen, 1990) have a duration of $381 \mu$ s such that they effectively invert the ${ }^{13} \mathrm{C}$ chemical shift range from 18 to $68 \mathrm{ppm}$. The ${ }^{15} \mathrm{~N}$ carrier is set to $116.5 \mathrm{ppm}$ and ${ }^{15} \mathrm{~N}$ high power pulses are applied at an rf field strength of $6.3 \mathrm{kHz}$, whereas Waltz decoupling during acquisition is carried out at an rf field strength of $1.3 \mathrm{kHz}$. Delay durations: $T_{\mathrm{LG}}=60 \mathrm{~ms} ; \delta=9.3 \mathrm{~ms} ; T=T_{\mathrm{LG}} / 4-\delta / 2$ (neglecting pulse lengths and switching delays); $\tau=1.4 \mathrm{~ms}$. Phase cycling: $\phi_{1}=2 x, 2(-\mathrm{x}) ; \phi_{2}=x,-x ; \phi_{3}=-y ; \phi_{4}=4 x, 4(-x) ;$ receiver $=x$, $2(-x), x,-x, 2(\mathrm{x}),-x$. Quadrature detection in the $t_{1}$ - and $t_{2}$-dimensions is obtained by altering $\phi_{2}$ and $\phi_{4}$ in the States-TPPI manner, respectively. Gradients (sine bell shaped; $25 \mathrm{G} / \mathrm{cm}$ at center): $\mathrm{G}_{1,2,3,4}=3(z), 2(y), 0.6(z)$ and $0.4(x) \mathrm{ms}$ (direction). For the reference experiment (see text), no $t_{1}$-incrementation is carried out. The flip angle of the $\left(90^{\circ}-\theta_{\mathrm{m}}\right)$ pulses is changed to $90^{\circ}$ and the phase cycling is: $\phi_{1}=2 x, 2(-x) ; \phi_{2}=x,-x ; \phi_{3}=x ; \phi_{4}=4 x, 4(-x) ;$ receiver $=x, 2(-x), x$. Decoupling of ${ }^{1} J_{\mathrm{NC}^{\prime}}$-splittings during the ${ }^{15} \mathrm{~N}$ evolution period can be achieved by $180^{\circ}{ }^{13} \mathrm{C}$ carbonyl decoupling pulses applied at the centers of the $T-t_{2} / 4$ and $T+t_{2} / 4$ intervals (not shown). Since the experiment was carried out with a limited ${ }^{15} \mathrm{~N}$ acquisition time of $21.3 \mathrm{~ms}$, the ${ }^{1} J_{\mathrm{NC}^{\prime}}$-splitting is not resolved and the decoupling was omitted for simplicity of the pulse programming. The experiment can also be carried out for the detection of long-range ${ }^{1} \mathrm{H}^{\mathrm{N}}$ RDCs to ${ }^{13} \mathrm{C}$ carbonyl nuclei. In this case the ${ }^{13} \mathrm{C}$ carrier is set to $177 \mathrm{ppm}$, the excitation width of the $\mathrm{G} 3$ pulses is narrowed to the carbonyl region, and an additional $180^{\circ}$ decoupling pulse is applied on the ${ }^{13} \mathrm{C}^{\alpha}$ resonances in the center of the $t_{2}$-evolution period.

with intensities proportional to $\cos ^{2}\left(\pi K_{\mathrm{HC}} T_{\mathrm{LG}} /\right.$ $\sqrt{ } 3)$. The coupling constant $K_{\mathrm{HC}}$ can then be extracted from the ratio of the cross and reference peak intensities as described previously (Meier et al., 2003).

\section{Comparison to the conventional long-range ${ }^{1} H^{N}{ }^{13} C$ RDC experiment}

The PMLG long-range ${ }^{1} \mathrm{H}^{\mathrm{N}-}-{ }^{13} \mathrm{C}$ RDC was carried out on the phage-aligned sample of protein G using a ${ }^{1} \mathrm{H}^{\mathrm{N}} \rightarrow{ }^{13} \mathrm{C}$ PMLG transfer time $T_{\mathrm{LG}}$ of $60 \mathrm{~ms}$. Figure $3 \mathrm{~A}$ shows strips from the 3D data set taken at the amide positions of the consecutive amino acids E32 to D41, which are part of protein G's $\alpha$-helix. For comparison, a data set with identical ${ }^{1} \mathrm{H}^{\mathrm{N}},{ }^{15} \mathrm{~N}$, and ${ }^{13} \mathrm{C}$ acquisition parameters and identical total experimental time
$(60 \mathrm{~h})$ was acquired using the previous longrange $\mathrm{HCN}$ experiment without PMLG decoupling and a normal INEPT ${ }^{1} \mathrm{H}^{\mathrm{N}} \rightarrow{ }^{13} \mathrm{C}$ transfer time of $60 \mathrm{~ms} / \sqrt{3} \approx 34.6 \mathrm{~ms}$. Strips from this experiment for the same set of amino acids are shown in Figure $3 \mathrm{~b}$. The sensitivity gain afforded by the PMLG decoupling is clearly visible. For example, cross peaks $\mathrm{V} 34^{\mathrm{HN}} \rightarrow \mathrm{V} 34^{\mathrm{C} \beta}, \mathrm{F} 35^{\mathrm{HN}} \rightarrow$ $\mathrm{F} 35^{\mathrm{C} \alpha / \mathrm{C} \beta}, \quad \mathrm{K} 36^{\mathrm{HN}} \rightarrow \mathrm{F} 35^{\mathrm{C} \alpha}, \quad \mathrm{K} 36^{\mathrm{HN}} \rightarrow \mathrm{K} 36^{\mathrm{C} \alpha / \mathrm{C} \beta}$, $\mathrm{Q} 37^{\mathrm{HN}} \rightarrow \mathrm{K} 36^{\mathrm{C} \beta}, \quad \mathrm{Q} 37^{\mathrm{HN}} \rightarrow \mathrm{Q} 37^{\mathrm{C} \alpha}, \quad \mathrm{N} 40^{\mathrm{HN}} \rightarrow$ $\mathrm{A} 39^{\mathrm{C} \alpha}, \mathrm{N} 40^{\mathrm{HN}} \rightarrow \mathrm{N} 40^{\mathrm{C} \alpha}$, and $\mathrm{D} 41^{\mathrm{HN}} \rightarrow \mathrm{N} 40^{\mathrm{C} \alpha}$ are absent or barely detectable without PMLG decoupling, but have rather strong intensities after the removal of the proton residual dipolar coupling in the new PMLG experiment.

Obviously, the sensitivity gain is most pronounced for the amide protons of the $\alpha$-helix (residues 28 to 41 ), which are most affected by 


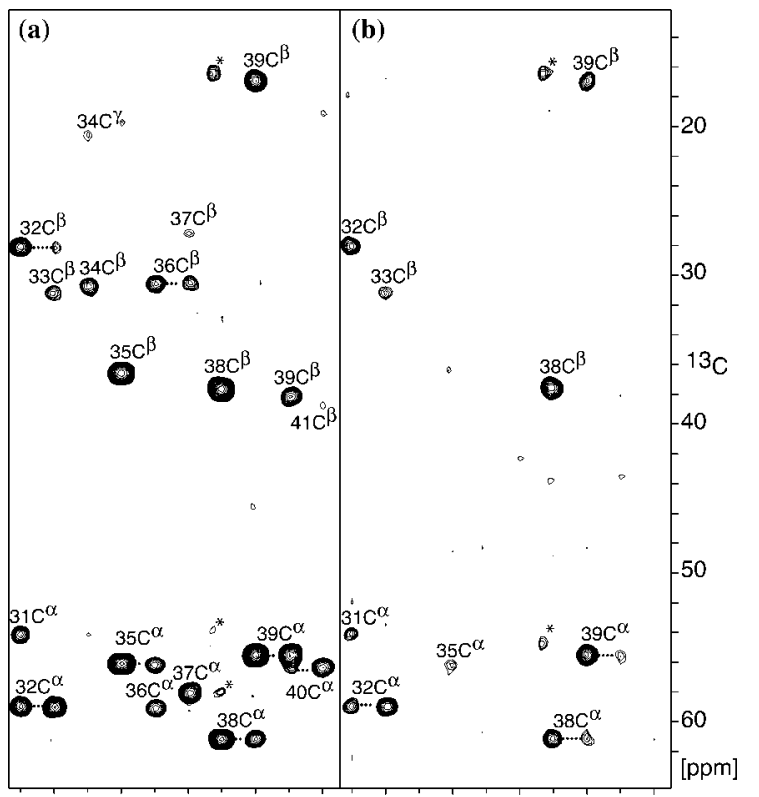

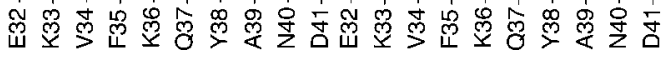

Figure 3. Comparison of PMLG (a) and conventional (b) longrange quantitative- $J \mathrm{HCN}$ experiments carried out on a sample of phage-oriented, ${ }^{15} \mathrm{~N} /{ }^{13} \mathrm{C} /{ }^{2} \mathrm{H}$-labeled (amide protonated) protein $\mathrm{G}$. The panels show ${ }^{1} \mathrm{H}^{\mathrm{N}}-{ }^{13} \mathrm{C}$ strips for the consecutive stretch of amino acids E32 to D41 extracted from the respective three-dimensional data sets acquired under identical conditions. Data points are marked with assignment information. Asterisks mark cross peaks resulting from overlap with adjacent amide resonances. In both experiments, data matrices were collected as $70 *\left({ }^{13} \mathrm{C}\right) \times 38 *\left({ }^{15} \mathrm{~N}\right) \times 768 *\left({ }^{1} \mathrm{H}^{\mathrm{N}}\right)$ complex data points with total acquisition times of $7.4\left({ }^{13} \mathrm{C}\right), 21.3\left({ }^{15} \mathrm{~N}\right)$, and $64\left({ }^{1} \mathrm{H}^{\mathrm{N}}\right) \mathrm{ms}$, respectively. The total experimental time for each experiment was $60 \mathrm{~h}$. (a) The experiment was carried out with PMLGdecoupled pulse sequence of Figure 2 with a total ${ }^{1} \mathrm{H}^{\mathrm{N}} \rightarrow{ }^{13} \mathrm{C}$ PMLG transfer time $T_{\mathrm{LG}}$ of $60 \mathrm{~ms}$. (b) The experiment was carried out as the conventional long-range HCN-HSQC (Meier et al., 2003) with a normal INEPT ${ }^{1} \mathrm{H}^{\mathrm{N}} \rightarrow{ }^{13} \mathrm{C}$ transfer time of $60 \mathrm{~ms} / \sqrt{ } 3 \approx 34.6 \mathrm{~ms}$.

strong residual dipolar couplings to protons. However, sensitivity gains are also achieved in other regions of the protein. Figure 4 shows a histogram of the amplitude ratios of all crosspeaks detected in the experiments with and without PMLG decoupling. As an average over all detected crosspeaks, the relative sensitivity increase of the PMLG experiment is 2.4 , whereas average gains of 3.7 and 1.3 are obtained in the $\alpha$-helical region and the flexible $\mathrm{C}$-terminal part, respectively. Overall, $170{ }^{1} \mathrm{H}^{\mathrm{N}}{ }^{13} \mathrm{C}^{\text {aliphatic }}$ couplings could be determined from the PMLG-HCN experiment optimized for the transfer to aliphatic carbon nuclei, as compared to 133 couplings of the conventional HCN experi-

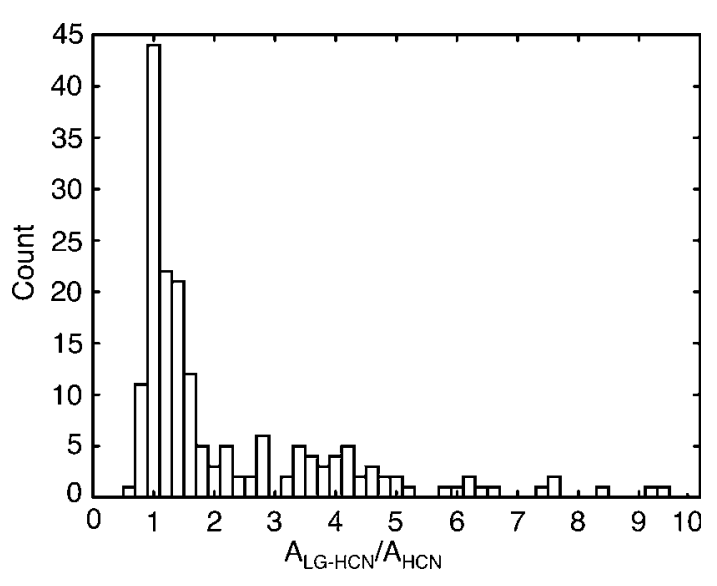

Figure 4. Sensitivity gain of the PMLG over the conventional long-range quantitative- $J$ HCN experiment. The relative amplitudes of the cross peaks of the two experiments $A_{\mathrm{LG}-\mathrm{HCN}} /$ $A_{\mathrm{HCN}}$ are shown as a histogram. Amplitudes are derived from the data sets described in Figure 3. For correlations that were only detectable in the PMLG, but not in the conventional HCN experiment, the amplitudes of the conventional experiment were set to three times the rms noise.

ment carried out under identical conditions. The maximal distance detected by the aliphatic PMLG-HCN experiment is $4.9 \AA$ for the atom pair $\mathrm{L} 10^{\mathrm{HN}} / \mathrm{V} 21^{\mathrm{C} \beta}$, as judged from the coordinates of the $1.1 \AA$ crystal structure of protein $G$ (1IGD)(Derrick and Wigley, 1994) after the addition of amide hydrogen positions by the program MOLMOL (Koradi et al., 1996). Similar to the original HCN experiment (Meier et al., 2003), the PMLG HCN can also be carried out for the detection of long-range RDCs from amide protons to carbonyl ${ }^{13} \mathrm{C}$ nuclei by simple adjustments of the ${ }^{13} \mathrm{C}$ excitation profiles (see legend to Figure 2). Such a long-range ${ }^{1} \mathrm{H}^{\mathrm{N}}{ }^{13} \mathrm{C}^{\text {carbonyl }}$ PMLG-HCN experiment gives similar sensitivity enhancements as the present ${ }^{1} \mathrm{H}^{\mathrm{N}}-{ }^{13} \mathrm{C}^{\text {aliphatic }}$ PMLG-HCN (data not shown).

In order to derive residual ${ }^{1} \mathrm{H}^{\mathrm{N}}-{ }^{13} \mathrm{C}$ dipolar couplings $D_{\mathrm{HC}}$ from the measured total coupling constants $K_{\mathrm{HC}}=J_{\mathrm{HC}}+D_{\mathrm{HC}}$, a second quantitative PMLG-HCN-experiment was carried out on an isotropic sample of protein $\mathrm{G}$, which yielded the scalar coupling constants $J_{\mathrm{HC}}$. The dipolar couplings $D_{\mathrm{HC}}$ were then calculated as the difference of the two measurements. The accuracy of the determined residual dipolar couplings was judged from a comparison to dipolar couplings predicted from the 1IGD crystal structure and the orientation tensor determined 


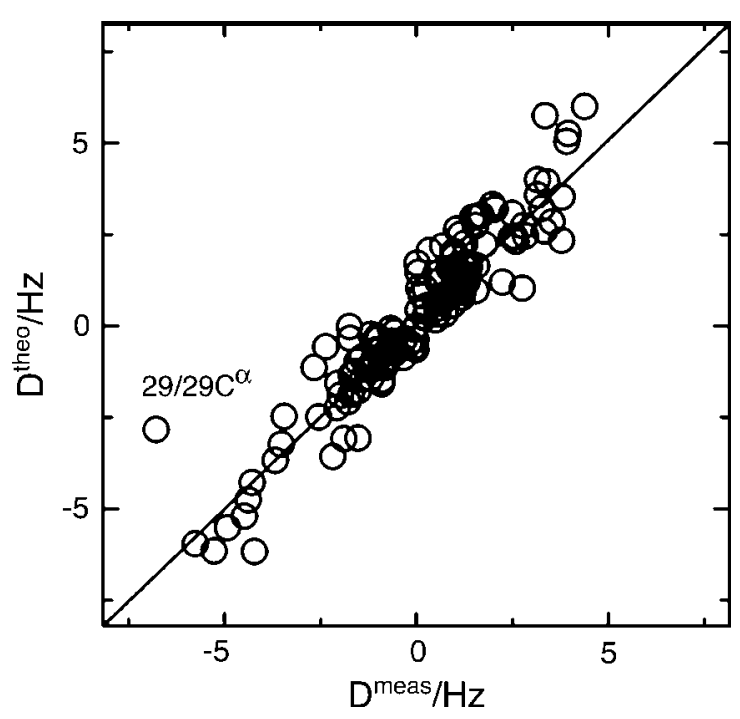

Figure 5. Comparison of measured and predicted long-range ${ }^{1} \mathrm{H}^{\mathrm{N}}-{ }^{13} \mathrm{C}$ RDCs for phage-oriented protein G. Measured RDCs $\left(D^{\text {meas }}\right)$ were derived as the difference of ${ }^{1} \mathrm{H}^{\mathrm{N}}-{ }^{13} \mathrm{C}$ couplings obtained by the PMLG HCN experiment on the phage-oriented and the isotropic protein $\mathrm{G}$ sample. Predicted RDCs $\left(D^{\text {theo }}\right)$ were calculated according to the 1IGD crystal structure and an orientation tensor determined from an independent set of ${ }^{1} D_{\mathrm{HN}}$ RDCs. Ambiguities in the experimental RDCs resulting from the unknown sign of dipolar and scalar couplings were resolved according to the best fit to the predicted data. An outlier RDC $\left(\mathrm{A} 29^{\mathrm{HN}} \rightarrow \mathrm{A} 29^{\mathrm{C} \alpha}\right)$ at the position of a point mutation in our construct (E29A) is indicated by assignment information.

from a measurement of ${ }^{1} D_{\mathrm{HN}}$ RDCs (Figure 5). For the total number of the detected 170 ${ }^{1} \mathrm{H}^{\mathrm{N}}{ }^{13} \mathrm{C}^{\text {aliphatic }}$ the rms deviation between measured and predicted values is $0.9 \mathrm{~Hz}$, which is comparable to the rms deviation of the RDCs derived from the conventional $\mathrm{HCN}$ experiment $(0.8 \mathrm{~Hz})$. The corresponding quality factor $Q$ $\left(=\operatorname{rmsd}\left(D^{\text {measured }}-D^{\text {predicted }}\right) / \mathrm{rms}\left(D^{\text {measured }}\right)\right.$, (Cornilescu et al., 1998)) is 0.36. As noted previously (Meier et al., 2003), the deviations from the prediction are not distributed equally along the primary sequence of protein $\mathrm{G}$, but are most pronounced in loop and turn regions and at positions of mutated residues (e.g. E29A in Figure 5). This indicates that part of the deviations correspond to true differences in structure or structural order between the solution and the crystalline form of the protein. Therefore, the accuracy of individual long-range RDCs is larger than anticipated from the overall quality factor. From an analysis of the statistical and systematic errors of the conventional $\mathrm{HCN}$, it was estimated previously that the accuracy should be sufficient to define individual average internuclear distances to within few picometers (Meier et al., 2003).

\section{Summary}

In summary, we have explored the use of $\mathrm{rf}$ PMLG homonuclear dipolar decoupling for high resolution liquid state experiments of biomolecules under weak alignment conditions. The PMLG decoupling sequence can be incorporated efficiently into HMQC and INEPT modules such that considerable sensitivity gains are achieved during extended magnetization transfer periods. The use of the PMLG decoupling is expected to be most beneficial for weakly aligned small to medium-sized biomacromolecules where proton linewidths are limited by homonuclear dipolar truncation.

\section{Acknowledgements}

We thank Sebastian Meier for very helpful discussions and Marco Rogowski and Klara Rathgeb-Szabo for expert preparation of the isotope labeled protein. This work was supported by SNF grants 31-61'757.00 and 4049-069384.

\section{References}

Bax, A. (2003) Protein Sci., 12, 1-16.

Bielecki, A., Kolbert, A.C. and Levitt, M.H. (1989) Chem. Phys. Lett., 155, 341-346.

Boisbouvier, J., Delaglio, F. and Bax, A. (2003) Proc. Natl. Acad. Sci. USA, 100, 11333-11338. Epub 2003 Sep 12.

Cornilescu, G., Marquardt, J.L., Ottiger, M. and Bax, A. (1998) J. Am. Chem. Soc., 120, 6836-6837.

Delaglio, F., Grzesiek, S., Vuister, G.W., Zhu, G., Pfeifer, J. and Bax, A. (1995) J. Biomol. NMR, 6, 277-293.

Derrick, J.P. and Wigley, D.B. (1994) J. Mol. Biol., 243, 906 918.

Emsley, L. and Bodenhausen, G. (1990) Chem. Phys. Lett., 165, 469-476.

Hansen, M.R., Mueller, L. and Pardi, A. (1998) Nat. Struct. Biol., 5, 1065-1074.

Koradi, R., Billeter, M. and Wüthrich, K. (1996) J. Mol. Graph., 14, 51-55, 29-32.

Lee, M. and Goldburg, W.I. (1965) Phys. Rev., 140, 1261-1271.

LeMaster, D.M. and Richards, F.M. (1988) Biochemistry, 27, $142-150$.

Marjanska, M., Castiglione, F., Walls, J.D. and Pines, A. (2002) J. Magn. Reson., 158, 52-59.

Mehring, M. and Waugh, J.S. (1972) Phys. Rev. B, 5, 34593471 . 
Meier, S., Haussinger, D., Jensen, P., Rogowski, M. and Grzesiek, S. (2003) J. Am. Chem. Soc., 125, 44-45.

Piotto, M., Saudek, V. and Sklenar, V. (1992) J. Biomol. $N M R, 2,661-665$.

Tjandra, N. and Bax, A. (1997) Science, 278, 1111-1114.

Tolman, J.R., Flanagan, J.M., Kennedy, M.A. and Prestegard, J.H. (1995) Proc. Natl. Acad. Sci. USA, 92, 92799283 .
Torchia, D.A., Sparks, S.W. and Bax, A. (1988) J. Am. Chem. Soc., 110, 2320-2321.

Vega, A.J. (2004). J. Magn. Reson., 170, 22-41.

Vinogradov, E., Madhu, P.K. and Vega, S. (1999) Chem. Phys. Lett., 314, 443-450

Wu, Z. and Bax, A. (2002) J. Am. Chem Soc., 124, 96729673. 